\title{
Microphase Separation of Diblock Copolymer with Moving Walls
}

\author{
Antonio Daud Júnior, Flavio Moretti Morais, Sílvia Martins, \\ Faculdade de Física, Universidade Federal de Uberlândia, Uberlândia, MG, 38400-902, Brazil \\ Débora Coimbra, \\ Departamento de Física, Universidade Federal do Amazonas, Manaus, AM, 69077-000, Brazil \\ and Welles A. M. Morgado \\ Pontifícia Universidade Católica do Rio de Janeiro, Departamento de Física, Rio de Janeiro, RJ, 22452-970, Brazil
}

Received on 4 February, 2004

\begin{abstract}
Diblock copolymers are linear chain molecules consisting of two subchains A and B grafted covalently to each other. Below some critical temperature $T_{c}$ these two blocks tend to segregate, but due to the covalent bond they can segregate at best locally to form periodic structures (microdomains). For molecules whose subchains have the same length, the equilibrium pattern is lamellar. In the bulk regime, these microdomains are ordered at random. To obtain an oriented lamellar pattern it is necessary to consider some asymmetry. In the presence of an external field, the lamellae will align to it. Directional quenching also can lead to the growth of oriented microphase separation. The effect of boundary conditions (confinement between parallel walls) also generates well-aligned lamellae, parallel to the walls. If the distance between the walls is comparable to the molecular sizes, another constraint is imposed on the system since the domains are forced to accommodate between the walls and, for certain conditions we will see a frustration phenomenon. If we allow the walls to move with a certain velocity during phase segregation, the accommodation of the lamellae can be changed. We use a cell dynamical system, which is a very efficient computational method, in order to investigate the effect of moving walls in lamellae formation.
\end{abstract}

\section{Introduction}

Diblock copolymers are linear-chain molecules consisting of two subchains of different monomers A and B, bounded covalently to each other [1]. Below some critical temperature $T_{C}$, the two polymers species tend to separate, but due to the covalent bond they are unable to form only two domains, growing in a periodic structure which is locally segregated on the scale of nanometers, referred to microphase separation. If the two blocks have equal length, they segregate in a lamellar structure parallel to the walls of confinement, when this interaction is dominant.

Microphase separation has been widely studied during the last twenty years, for technological and theoretical reasons. As shown by Bates and Fredrickson [2], control over molecular scale morphology allows the development of new kinds of materials, of great utility for industry. From a fundamental viewpoint, films of polymers present advantageous model systems for investigations of phase equilibria in reduced geometry. Likewise, the influence of physical boundaries on the kinetics of phase separation and the resulting equilibrium morphology can be studied in quite some detail [3]. From the technological view, they are present in commercial products as coatings, paints, photoresists, and even exciting applications in lithography, because of self- organization of amphiphilic block copolymers [3].

Diblock copolymers films have been studied in a variety of settings: interacting walls either symmetric or asymmetric, neutral walls with matched densities $[4,5,6,7,8,9]$ and neutral walls confining molecules with mismatched densities [10] and gravity effects [11], to investigate pattern formation in a film confined between two hard surfaces. As for pattern formation, we can summarize all the above situations: when the walls are neutral, in the absence of mismatch, lamellae will form in the direction normal to the substrate; if interaction with the walls is added, the lamellae will form parallel to the walls; for molecules with density mismatch in the presence of a gravitational field normal to the confining walls, lamellae form parallel to the walls.

In this article we assume that the walls are not rigid, and we investigate the influence of moving walls on pattern formation. For this we simulate the diblock copolymer using the cell dynamics system (CDS) model [12]. Oono and Shiwa [13] first studied pattern formation in this model, which is intensively used nowadays. The spirit of their modeling was purely phenomenological [14], however the motivation was to have the simplest model to give a spatially nonuniform equilibrium pattern. CDS consists in a map that describes the order parameter evolution of each little portion (cell) of the system in a mesoscopic scale. It is a 
discreet formulation able to describe on a non-continuous functional, computationally efficient to the numerical study of phase ordering [15]. This work is organized as follows: in the next section we present the basic formulation of CDS model and our considerations to include the effect of moving walls. Then, in the third section, we discuss the results obtained. Our conclusions are presented in the final section.

\section{CDS Model}

The CDS model is a map that sends an instantaneous discrete frame of spatial pattern to another near it. We assign a scalar variable $\psi(n, t)$ to each lattice site corresponding to the coarse-grained order parameter in the $n$th cell at time $t$ (time here is defined as the number of interations). This order parameter represents the difference $\psi_{A}-\psi_{B}$, where $\psi_{A}\left(\psi_{B}\right)$ is the local number density of $A(B)$ species. First, we consider the local dynamics (each cell), analyzing if the order parameter increases or not. Second, we include the nearest neighbor interaction. Next, we must impose the condition of conservation of order parameter and connectivity of distinct species. Taking this to account, we can write

$$
\begin{aligned}
\psi(n, t+1)= & (1-\epsilon) \psi(n, t)+ \\
& \langle\langle C(i, j ; \operatorname{sgn}[\alpha]) \times \alpha\rangle\rangle,
\end{aligned}
$$

where $\alpha=[I(n, t)-I(j, t)]$ and

$$
\begin{aligned}
I(n, t)= & D \tanh [\psi(n, t)]-\psi(n, t) \\
& +E(\langle\langle\psi(n, t)\rangle\rangle-\psi(n, t))
\end{aligned}
$$

is the chemical potential; $\langle\langle\rangle\rangle$ represents the isotropic space average and $\epsilon, D$ and $E$ are positive phenomenological constants. The parameter $\epsilon$ appears in this model to stabilize the solution $\psi=0$ in the bulk. Also,

$$
C(i, j ; \alpha)=\left[\psi_{c}+\alpha \psi(j)\right]\left[\psi_{c}-\alpha \psi(i)\right] / \psi_{c}^{2},
$$

where $\pm \psi_{c}$ denote the zeroes of $D \tanh [\psi(n, t)]-\psi(n, t)$.

The DCS formulation is equivalent to a discrete formulation of the Cahn-Hilliard equation, usually employed to study polymer phase separation. The former has the advantage that the function $\tanh [\psi(n, t)]$ is more stable than the cubic polynomial form of the Cahn-Hilliard order parameter [1].

The movement of the walls and the interaction of the subchain with the surface are considered in the boundary conditions. The intensity of the interaction with the surface is given by the parameter $\sigma$, and we assume that each surface attracts a different subchain.

\section{Results and Discussion}

For the simulations we choose a $25 \times 256$ initial lattice, $A=1.2, D=0.5, \epsilon=0.01$, and a uniformly distributed initial condition. At each time step, we impose the movement of the walls, with a stochastic velocity,

$$
v=\left\langle\frac{\sum \Delta x_{i}}{t}\right\rangle \alpha P
$$

where $\Delta x_{i}=0$ or 1 with the probability $P$ and $t$ is the computational time (number of interactions). A schematic view can be seen in Fig. 1.
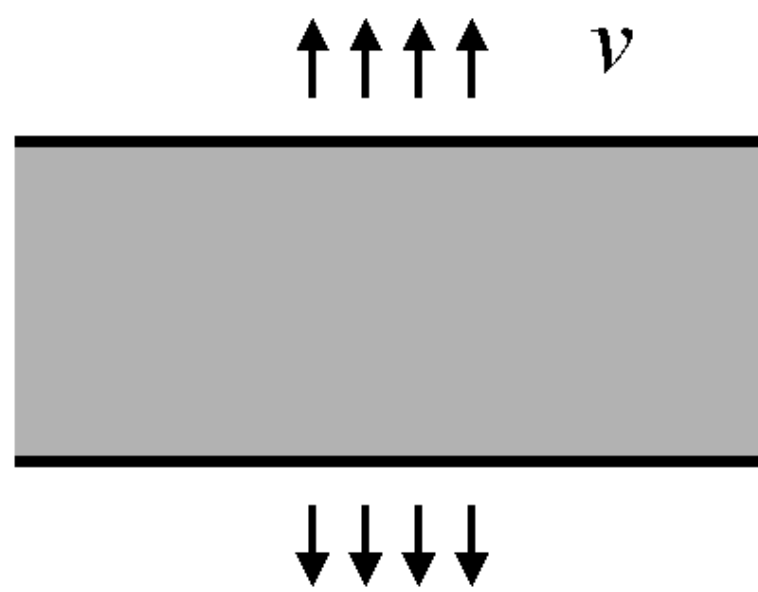

Figure 1. Schematic view of the movement of the wall.

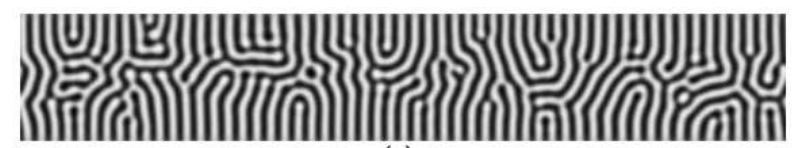

(a)

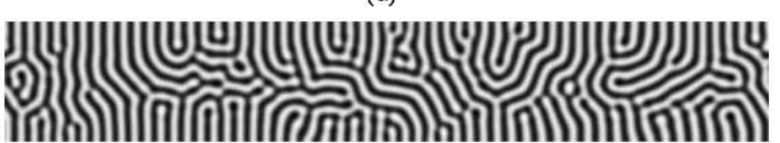

(b)

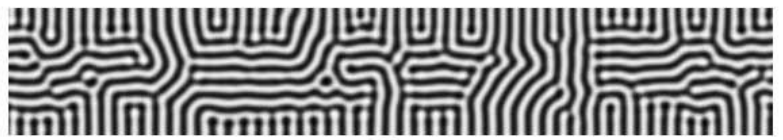

(c)

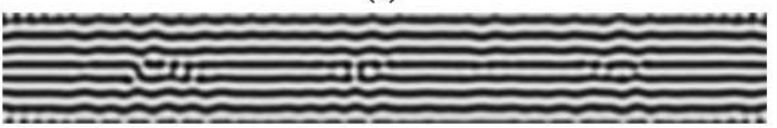

(d)

Figure 2. For all patterns, $P=0.005$. (a) $\sigma=0$, (b) $\sigma=0.0001$, (c) $\sigma=0.001$, (d) $\sigma=0.01$.

In Fig. 2, we can see the effect of the surface interaction on the pattern formation, after 2000 iterations. For larger values of $\sigma$, the tendency of the pattern is to form lamellae parallel to the surface. When $\sigma$ is small, the movement of the walls leads to the formation of perpendicular lamellae.

In Fig. 3 the time evolution of the pattern is shown for parameter values $P=0.01$ and $\sigma=0.01$. We now see the competition between the two effects: the pattern exhibits both parallel and perpendicular lamellae. The size of lamellae, $w$, changes slowly, as can be seen in Fig. 4. To accommodate the pattern between the walls, the number of lamellae changes, growing with time. 


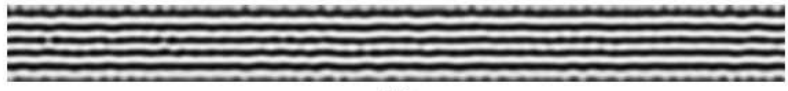

(a)

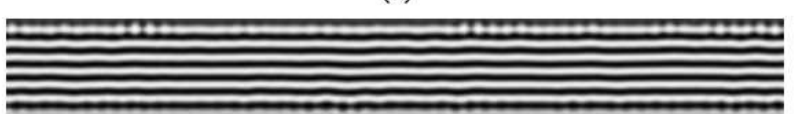

(b)

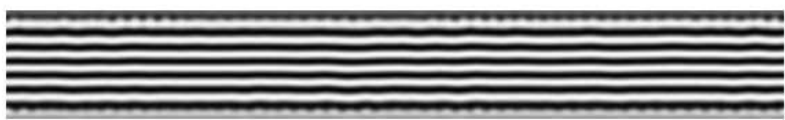

(c)

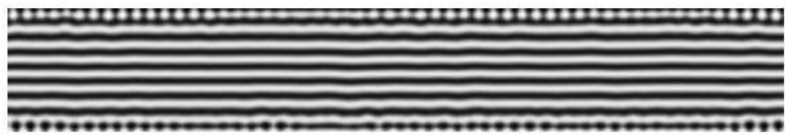

(d)
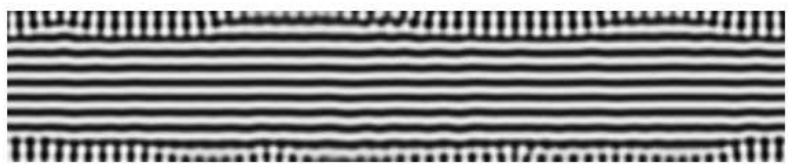

(e)

Figure 3. For all patterns, $\sigma=0.01$ and $P=0.01$. (a) $t=500$, (b) $t=1000$, (c) $t=1500$, (d) $t=2000$, (e) $t=2500$.

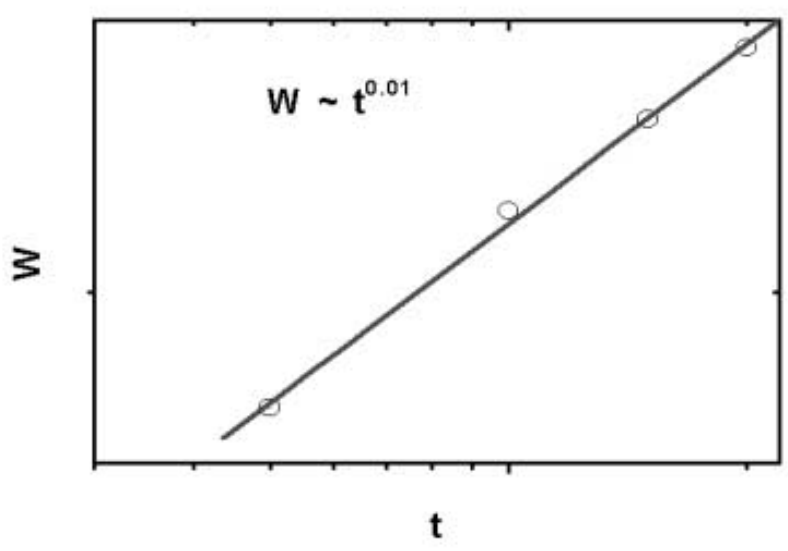

Figure 4. Lamellae size evolution.

\section{Final Remarks}

In this work, we applied a cell dynamical system computational method to investigate the effect of moving walls on lamellae formation of diblock copolymers. The effect of confinement between parallel walls, simulated in boundary conditions, generated well-aligned lamellae, parallel to the walls for larger values of the surface interaction $\sigma$. For small $\sigma$, the movement of the walls provokes formation of perpendicular lamellae. When the distance between the walls is comparable to the molecular sizes, the domains are forced to accommodate between the walls and, for certain conditions, we observe frustration. If we allow the walls to move during phase segregation, the accommodation of the lamellae changes. Our results also demonstrate a slow time evolution of the size of lamellae due to competition between the stochastic velocity of movement and the surface interaction. In this case the pattern exhibits both parallel and perpendicular lamellae.

\section{References}

[1] W. A. M. Morgado, S. Martins, M. Bahiana, M. S. O. Massunaga, Comp. Phys. Comm. 121/122, 327 (1999).

[2] F. S. Bates and G. H. Fredrickson, Phys. Today, 52 (1999).

[3] H. Elbes, K. Fukunaga, R. Stadler et al., Macromolecules 32, 1204 (1999).

[4] G. T. Pickett, A. C. Balazs, Macromolecules 30, 3097 (1997).

[5] G. Brown, A. Chakrabarti, J. Chem. Phys. 102, 3310 (1995).

[6] M. S. Turner, Phys. Rev. Lett. 69, 1788 (1992).

[7] A. Menelle, T. P. Russel, S. Anastasiadis, Phys. Rev. Lett. 68, 67 (1992).

[8] P. Lambooy, T. P. Russel, G. J. Kellogg et al., Phys. Rev. Lett. 72, 2899 (1994).

[9] G. J. Kellogg, D. G. Walton, A. M. Mayes et al. Phys. Rev. Lett. 762503 (1996).

[10] W. A. M. Morgado, S. Martins, M. Bahiana, M. S. O. Massunaga, Phys. Rev. E 61, 4118 (2000).

[11] W. A. M. Morgado, S. Martins, M. Bahiana, M. S. O. Massunaga, Physica A 283, 208 (2000).

[12] K. Kitahara, Y. Oono, D. Jasnow, Mod. Phys. Lett. B 2, 765 (1988).

[13] Y. Oono, Y. Shiwa, Mod. Phys. Lett. B 1, 49 (1987).

[14] Y. Oono, M. Bahiana, Phys. Rev. Lett. 61, 1109 (1988).

[15] I. W. Hamley, Macromolecules 36, 9 (2000) 